\title{
Recent advances in melanin-like nanomaterials in biomedical applications: a mini review
}

\author{
Jihyo Park ${ }^{\dagger}$, Haeram Moon ${ }^{\dagger}$ and Seonki Hong ${ }^{*}$ (D)
}

\begin{abstract}
Background: Melanins are a group of biopigments in microorganisms that generate a wide range of colorants. Due to their multifunctionality, including ultraviolet protection, radical scavenging, and photothermal conversion, in addition to their intrinsic biocompatibility, natural melanins and synthetic melanin-like nanomaterials have been suggested as novel nano-bio platforms in biomedical applications.

Main body: Recent approaches in the synthesis of melanin-like nanomaterials and their biomedical applications have briefly been reviewed. Melanin-like nanomaterials have been suggested as endogenous chromophores for photoacoustic imaging and radical scavengers for the treatment of inflammatory diseases. The photothermal conversion ability of these materials under near-infrared irradiation allows hyperthermia-mediated cancer treatments, and their intrinsic fluorescence can be an indicator in biosensing applications. Furthermore, catecholrich melanin and melanin-like nanomaterials possess a versatile affinity for various functional organic and inorganic additives, allowing the design of multifunctional hybrid nanomaterials that expand their range of applications in bioimaging, therapy, theranostics, and biosensing.

Conclusion: Melanin-like natural and synthetic nanomaterials have emerged; however, the under-elucidated chemical structures of these materials are still a major obstacle to the construction of novel nanomaterials through bottom-up approaches and tuning the material properties at the molecular level. Further advancements in melaninbased medical applications can be achieved with the incorporation of next-generation chemical and molecular analytical tools.
\end{abstract}

Keywords: Melanin, Polydopamine, Bioimaging, Theranostics, Drug delivery, Biosensing

\section{Introduction}

Melanin refers to a group of biopigments in microorganisms, plants and animals that generates a wide range of colors from yellow to red (known as pheomelanin) and brown to black (called eumelanin). Melanin determines hair, eye, and skin color in humans and acts as a protective shield by adsorbing harmful ultraviolet (UV) light from solar radiation and scavenging reactive oxygen species (ROS) to prevent DNA damage to nearby cells [1]. Adsorbed solar energy can be dissipated as heat, contributing to the thermoregulation of animals, which is especially important for cold-blooded animals [2]. In the

\footnotetext{
* Correspondence: seonkihong@dgist.ac.kr

${ }^{\dagger}$ Jihyo Park and Haeram Moon contributed equally to this work.

Department of Emerging Materials Science, DGIST, Daegu 42988, South Korea
}

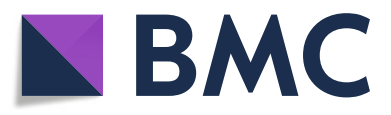

(c) The Author(s). 2019 Open Access This article is distributed under the terms of the Creative Commons Attribution 4.0 International License (http://creativecommons.org/licenses/by/4.0/), which permits unrestricted use, distribution, and

reproduction in any medium, provided you give appropriate credit to the original author(s) and the source, provide a link to the Creative Commons license, and indicate if changes were made. The Creative Commons Public Domain Dedication waiver (http://creativecommons.org/publicdomain/zero/1.0/) applies to the data made available in this article, unless otherwise stated.

brain, neuromelanin is produced in some populations of catecholaminergic neurons with uncertain circumstances, with attention due to its hypothetical relationship to neurodegenerative disorders such as Parkinson's disease $[3,4]$. Melanin also plays a major role in the innate immune system of insects against bacterial and fungal pathogens [5]. Its multifunctionality and intrinsic biocompatibility have inspired scientists to develop novel nanomaterials by mimicking its chemical structure and material properties.

Among various precursors, dopamine (DA) has been a major precursor in the in vitro synthesis of melanin-like nanomaterials because it can be nonenzymatically crosslinked to 5,6-dihydroxyindole (DHI) under relatively moderate conditions, such as mildly basic $\mathrm{pH}(\sim 8.5)$ and/or in the presence of dissolved oxygen in aqueous 
reaction solutions (details will be discussed in chapter 2.1). In addition to melanin-like properties, the versatile adhesive properties of DA-derived melanin (also called polydopamine, pDA) has allowed limitless applications in biomedical and environmental fields since it was first reported in 2007 [6]. pDA was first synthesized as a material-independent surface coating material that mimics the chemical composition of mussel adhesive proteins, and it has also been suggested as a synthetic model of natural melanin, in particular neuromelanin in the brain, to elucidate the unexplored mechanisms of natural melanin in nature [7]. pDA possesses various functional groups, such as protonated and/or unprotonated amine groups, catechols, and dihydroxyindole rings, that participate in multimodal molecular interactions on the surface of various types of materials constructing multifunctional coatings and adsorbing a bunch of molecules and ions as adsorbents (details will be discussed in chapter 2.2).

Here, we briefly review recent advances in natural melanin and melanin-like nanomaterials, such as $\mathrm{pDA}$, that have been established for biomedical applications. Their synthesis and fabrication into nanoparticles, hollow nano/microcapsules, and core@shell structures with a controlled size and shape will be summarized in chapter 2 , and their emerging applications in bioimaging, theranostics, sensors, and drug delivery systems will be introduced in chapter 3 .

\section{Synthesis and characterization of melanin-based multifunctional nanomaterials}

\section{Synthesis of artificial melanin}

Melanogenesis in nature is initiated through the activation of tyrosinase, laccase and other polyphenol oxidases in response to external stimuli such as chemical stresses (metal ions, ROS, oxidizing agents), UV exposure, and pathogenic attack (particularly in insects) [8]. These enzymes convert the precursors (tyrosine, DOPA, and DA) into major intermediates, DHI and/or 5,6-dihydroxyindole-2-carboxylic acid (DHICA), that can be further polymerized and assembled into melanin granules. Inspired by biosynthesis in nature, the synthesis of melanin-like nanomaterials has been achieved with natural precursors as well as their synthetic analogs crosslinked via enzymes in vitro. $\mathrm{F}$. Li et al. reported that pDA nanoparticles synthesized by laccase were more stable in strongly acidic and alkaline solutions than the chemically synthesized nanoparticles [9]. A. Lampel et al. systematically demonstrated the relationship between the structural variation of precursors and the resulting melanin-like material properties by tuning the amino acid sequence of selfassembled tripeptide crystalline precursors containing phenylalanine, aspartic acid, and tyrosine residues [10]. Enzymatic polymerization via tyrosinase not only formed covalent crosslinking between tyrosine residues but also altered noncovalent interactions in preordered crystalline tripeptides, resulting in a wide range of disordered melanin-like pigments synthesized. Surface-immobilized enzymes have allowed for the site-specific deposition of melanin-like nanoparticles in proximity to enzymes; however, the synthesis was self-terminated due to the uncontrolled adhesion of the synthesized particles to enzymes [11].

Chemical oxidation processes can be the ultimate alternatives to the enzymatic synthesis of melanin-like nanomaterials that allows for cost-effective mass production with reproducibility. Melanin-like pDA nanoparticles have been synthesized with tunable sizes ranging from a few nanometers to hundreds of nanometers in aqueous solution at mildly basic $\mathrm{pH}$ values by the addition of different ratios of sodium hydroxide $(\mathrm{NaOH})$ or ammonium hydroxide $\left(\mathrm{NH}_{4} \mathrm{OH}\right)$ to DA. During these processes, X. Wang reported that radicals could further control the growth of the particles by either quenching the radical intermediates of $\mathrm{pDA}$ or accelerating seed formation [12]. Particle size was also affected by buffers; N. F. D. Vecchia et al. reported that the particle size of pDA increased with increasing initial DA concentration in phosphate or bicarbonate buffers, but inhibition of particle growth was observed in Tris buffer [13]. The authors suggested that the covalent attachment of Tris molecules to the surface of the growing pDA surface prevented particle growth. X. Jiang et al. confirmed the effect of the water-alcohol co-solvent system on pDA synthesis via an ammonia solution and established an empirical formula that predicts the size of the resulting particles in mixed solvents [14]. Chemical oxidants such as $\mathrm{KMnO}_{4}, \mathrm{NaIO}_{4}$, and $\mathrm{CuSO}_{4}$, and organic bases such as piperidine, have also been used for the synthesis of melanin-like particles and coatings in acidic $\mathrm{pH}$ conditions or in organic solvents [15-17]. Finally, temperature is another factor that can control the size of the growing particles [18].

Supramolecular assembly through noncovalent interactions has been suggested to be critical in regulating the shape and properties of melanin-like nanomaterials in addition to covalent crosslinking [19]. Theoretical and experimental studies have suggested various types of noncovalent interactions, such as hydrogen bonding, van der Waals interactions, and $\pi-\pi$ stacking between oligomeric intermediates [20]. In 2012, Hong et al. experimentally isolated a trimer composed of DA and DHI assembled through unknown noncovalent interactions, suggesting that the building blocks for noncovalent assembly could be sufficiently low as oligomers, not polymers [21]. They subsequently reported experimental evidence in 2018 that cation- $\pi$ interactions can be the major driving force for the molecular assembly of 
oligomeric intermediates into granules [22]. In addition to experimental studies, computational methods suggested that large molecular weight oligomers are less likely to form, and tetramers can be notably more stable than the other forms, implying that the major building blocks for noncovalent assembly can be quite repetitive, although the resulting granules possess diversity in their material properties [23].

\section{Synthesis of melanin-like hybrid nanomaterials}

During both the enzymatic and chemical syntheses of melanin-like nanomaterials, additives can be simultaneously incorporated via either covalent crosslinking to growing oligomers or physically entrapped in particles through noncovalent interactions to tune their material properties (as summarized in Table 1). For example, fluorescent pDA nanoparticles were obtained in the presence of glutathione (GSH) as an additive to a DA precursor with potential applications in biosensing [47]. Ferric ion-complexed pDA nanoparticles were synthesized in one-pot and further annealed at $650{ }^{\circ} \mathrm{C}$ for $3 \mathrm{~h}$, resulting in carbonized pDA embedded with $3-5 \mathrm{~nm}$ sized $\mathrm{Fe}_{3} \mathrm{O}_{4}$ as an organic/inorganic hybrid nanocatalyst [48]. A recent study by D. Wang et al. showed that DA precursors participated in constructing a metal-organic framework (MOF) with $\mathrm{Mn}^{2+}$ and organic linkers and further polymerized as a part of a MOF crystal, showing great potential for photothermal therapy (PTT) [49]. Hong et al. reported that cations such as the quaternary ammonium cation and potassium ions showed a remarkable affinity to building blocks of pDA via cation $-\pi$ interactions, generating superhydrophilicity in one-pot synthesized coatings [22]. Targeting moieties as well as therapeutic agents could also be incorporated

Table 1 A summary of melanin-like nanomaterials synthesized for biomedical applications

\begin{tabular}{|c|c|c|c|c|c|}
\hline \multicolumn{2}{|c|}{ Biomedical applications } & \multirow{2}{*}{$\begin{array}{l}\text { Template } \\
\text { pDA NP }\end{array}$} & \multirow{2}{*}{$\frac{\text { Size }}{80 \mathrm{~nm}}$} & \multirow{2}{*}{$\begin{array}{l}\text { Functional additives } \\
-\end{array}$} & \multirow{2}{*}{$\frac{\text { Ref }}{[24]}$} \\
\hline Therapy & ROS scavenger & & & & \\
\hline & & & $160 \mathrm{~nm}$ & $\mathrm{w} /$ and $\mathrm{w} / \mathrm{O} \mathrm{Cu}^{2+}$ & [25] \\
\hline & $\begin{array}{l}\text { Photodynamic } \\
\text { therapy }\end{array}$ & pDA/PEG coating on $\mathrm{CaCO}_{3}$ & $40 \mathrm{~nm}$ & Photosensitizer: chlorin e6 & [26] \\
\hline & Photothermal & pDA NP & $160 \mathrm{~nm}$ & - & [27] \\
\hline & & PEGylated pDA NP & $78 \mathrm{~nm}$ & $\begin{array}{l}\text { Anticancer drug: doxorubicin, } \\
\text { 7-ethyl-10-hydroxycamptothecin }\end{array}$ & [28] \\
\hline & Drug delivery & pDA NP & $75-400 \mathrm{~nm}$ & Anticancer drug: camptothecin & [29] \\
\hline & system & PEGylated pDA NP & $28 \mathrm{~nm}$ & $\begin{array}{l}\text { Ligand: triphenylphosphonium } \\
\text { Anticancer drug: doxorubicin }\end{array}$ & [30] \\
\hline & & $\begin{array}{l}\text { Polyethylenimine-decorated } \\
\text { pDA NP }\end{array}$ & $60-80$ nm & Therapeutics: plasmid DNA & [31] \\
\hline & & $\begin{array}{l}\text { Poly (methacrylic acid) } \\
\text {-coated pDA capsule }\end{array}$ & 300 nm & Anticancer drug: doxorubicin & [32] \\
\hline & & pDA coating on insulin NP & $50,71,84 \mathrm{~nm}$ (coating) & - & [33] \\
\hline & & $\begin{array}{l}\text { pDA/PEG coating on } \\
\text { mesoporous silica NP }\end{array}$ & $193.08 \mathrm{~nm}$ & $\begin{array}{l}\text { Ligand: folic acidAnticancer } \\
\text { drug: doxorubicin }\end{array}$ & [34] \\
\hline \multirow[t]{8}{*}{$\begin{array}{l}\text { Bioimaging and } \\
\text { theranostics }\end{array}$} & $\begin{array}{l}\text { Photoacoustic } \\
\text { Imaging }\end{array}$ & $\begin{array}{l}\text { Ethylenediamine/PEG-coated } \\
\text { pDA NP }\end{array}$ & $130 \mathrm{~nm}$ & - & [35] \\
\hline & & pDA-coated AuNP & $150 \mathrm{~nm}$ & - & [36] \\
\hline & & PDA-coated $\mathrm{Fe}_{3} \mathrm{O}_{4} \mathrm{NP}$ & $15 \mathrm{~nm}$ & ${ }^{64} \mathrm{Cu}$ & {$[37]$} \\
\hline & & Melanin NP & $42.5 \pm 2.1 \mathrm{~nm}$ & Targeting moiety: poly-L-lysine & [38] \\
\hline & Magnetic resonance & pDA NP & $51,119,188 \mathrm{~nm}$ & Cu (II) & [39] \\
\hline & & PEGylated pDA NP & $70-300$ nm & $\mathrm{Mn}(I I)$ & [40] \\
\hline & & & $25-43 \mathrm{~nm}$ & Fe (III) & [41] \\
\hline & & PEGylated Melanin NP & $13 \mathrm{~nm}$ & Gd (III), Cu (II) & [42] \\
\hline \multirow[t]{4}{*}{ Biosensing } & Fluorescent pDA & $\begin{array}{l}\text { pDA with } \\
\text { ethylenediamine NP }\end{array}$ & Tens of nm & - & [43] \\
\hline & & pDA NP reduced by $\mathrm{NaBH}_{4}$ & $34.83 \mathrm{~nm}$ & - & [44] \\
\hline & Fluorescence quencher & Mesoporous pDA NP & 70 nm & $\begin{array}{l}\text { Fluorescein amidites } \\
\text { (FAM)-labeled DNA probe }\end{array}$ & [45] \\
\hline & & pDA-coated upconversion NP & $5 \mathrm{~nm}$ (coating) & Cy3-aptamer & [46] \\
\hline
\end{tabular}


into growing melanin-like nanoparticles, with potential in bioimaging and theranostics [50] (details are shown in chapter 3.3).

As-prepared melanin-like nanoparticles can be chemically reactive core templates for multifunctional core@shell nanostructures. Metal ions can chelate to the surface of nanoparticles for bioimaging, such as in photoacoustic imaging (PAI) [42] and magnetic resonance imaging (MRI) [40, 41]. Silver ions could be adsorbed and simultaneously reduced on the surface of artificial melanin, allowing for the green synthesis of reductant-free organic/inorganic hybrid nanoparticles with antibacterial activity [51]. Nucleophiles such as thiols and amines are commonly used moieties in immobilizing organic molecules on the surface of asprepared melanin-like nanoparticles without the additional chemical reagents [6]. Thiolated polyethylene glycol could be used for the surface PEGylation of melanin-like nanoparticles to enhance the biocompatibility and stability in vivo [28, 35]. Targeting moieties such as RGD, folic acid, and triphenylphosphonium (TPP) could be immobilized on the surface of nanoparticles with anticancer drugs such as doxorubicin (DOX) and camptothecin (CPT) for tumor-targeting therapeutic delivery $[29,52-55]$ (details are shown in chapter 3.2). Interestingly, tumor cell lysates adsorbed on pDA nanoparticles acted as an immune booster for vaccine development in colorectal cancer [56].

Melanin-like nanomaterials, and in particular pDA, could be coated on the surface of any kind of template/ core material to construct the reverse type of aforementioned core@shell nanostructures.pDA coating on the surface of inorganic nanoparticles enhances colloidal stability and prevents agglomerization and corrosion of inorganic nanoparticles [36, 37, 57-59]. In addition, it has been reported that pDA coating is not only biocompatible but also attenuates the intrinsic toxicity of inorganic materials such as quantum dots in vivo [60]. Melaninlike coating can also provide an adhesive nano-thin layer between the core and outer materials, expanding further design of theranostic nanomaterials with multifunctionality [61-64]. Moreover, by further modification of these processes, hollow melanin nanocapsules could be synthesized by dissolving sacrificial core materials of asprepared core@melanin hybrid nanoparticles [26].

\section{Biocompatibility and biostability of melanin-like multifunctional nanomaterials}

As a great candidate for biomedical applications, one of the strengths of melanin-like nanomaterials is their biocompatibility and long-term stability. They are not only well-dispersed in aqueous buffered medium, but also remained stable when stored in those solution without notable physical changes for up to at least several weeks
$[27,59]$. Coating of them to inorganic nanoparticles improve the water dispersibility and colloidal stability without altering thickness of the modification for long period of time [59], allowing a variety of both in vitro and in vivo biomedical applications.

Dopamine causes oxidative stress to cells and is cytotoxic, but it has been experimentally proven that melanin-like nanomaterials like pDA possesses good biocompatibility. In vitro cytotoxicity has been evaluated in many different types of mammalian cell lines including HeLa, 4 T1, HepG2, and NIH3T3; the cell viability was retained greater than $90 \%$ after incubating with those nanoparticles for up to several days [27, 35, 59, 65]. Hong et al. reported that there is still nonnegligible amount of free dopamine that physically entrapped in synthesized pDA, but it is rarely released from pDA due to strong non-covalent interactions and therefore does not cause severe cytotoxicity [21]. In vivo studies have confirmed that the biocompatibility of those nanomaterials contribute to attenuate the intrinsic cytotoxicity of conventional biomaterials. Hong et al. reported that pDA coating significantly reduced the leukocyte population alternation caused by conventional CdSe core and ZnS-capped nanocrystals in blood after tail vein injection to mice [60]. In addition, it was reported to suppress the macrophage adhesion after 4 days and the foreign body giant cells (FBGCs) formation after 14 days from subcutaneous implantation of biodegradable poly (L-lactic acid) film in rats [60]. Similarly, Liu et al. investigated the long-term biocompatibility as well as ultra-stability of pDA coating in vivo; pDA-coated gold nanoparticles were mainly uptaken by the Kupffer cells in the liver, the organ with the highest accumulation, while they were uptaken by a variety of cells in the spleen, the organ with the second highest accumulation. They were intact within those cells for at least six weeks without causing notable histological toxicity in mice [59].

\section{Biomedical applications \\ Anti-inflammatory and photothermal therapy}

Melanin-like nanoparticles, and in particular pDA, have been suggested as therapeutics in the treatment of antiinflammatory diseases because of the abundant phenolic groups that serve as radical scavengers. Zhao et al. demonstrated that $80 \mathrm{~nm}$-sized pDA nanoparticles efficiently scavenged either $\mathrm{H}_{2} \mathrm{O}_{2}{ }^{-}$or lipopolysaccharide (LPS)-induced cellular ROS in vitro, and further study in murine acute peritonitis models confirmed the successful suppression of in vivo inflammation [24]. Similarly, Bao and his colleagues validated the antioxidant capacity of 160 $\mathrm{nm}$-sized pDA nanoparticles and provided potential applications in periodontal disease treatment to relieve oxidative stress without any side effects [25]. 
Abundant phenolic groups in melanin-like nanoparticles not only scavenge radical species but also quench photosensitizers such as chlorin e6 (Ce6). Photosensitizers generate ROS, including singlet oxygen species, to kill nearby cells upon light exposure in photodynamic therapy (PDT). The current drawback of conventional photosensitizers is their undesirable photoactivation [24]. To overcome this, Han and his colleagues developed complexed nanoparticles consisting of pDA and a photosensitizer conjugated to hyaluronic acid [66]. Since the synthesized nanoparticles release the photosensitizer from the quencher pDA only in response to the degradation of hyaluronic acid by the tumor-localized intracellular enzymes (e.g., hyaluronidase), tumor-specific photoactivation is allowed [66]. Similarly, Z. Dong et al. reported a calcium carbonatepDA composite hollow nanoparticle with the loaded photosensitizer Ce6 that is released from pDA and photoactivated only in an acidic tumor environment [26].

Natural melanin and melanin-like synthetic nanoparticles possess broadband adsorption from the UV to nearinfrared (NIR) range [67]. Therefore, they can provide efficient photothermal conversion upon NIR irradiation, which can be used in hyperthermia-mediated cancer treatments, also called PTT. The major advantage of PTT is the spatial control of light exposure for targetspecific treatment, but the cytotoxicity of currently used photosensitizers and the limited penetration of the light source into the skin are obstacles in the widening of its application [68]. Recent studies have suggested that melanin-like nanoparticles can be the ultimate photosensitizers used in PTT because of their biocompatibility and biodegradability in addition to their strong NIRreactive photothermal conversion efficiency. Liu et al. reported that melanin-like nanoparticles showed a photothermal conversion efficiency of $40 \%$ upon NIR irradiation with an $808 \mathrm{~nm}$ laser and did not cause longterm toxicity in rats in the absence of light exposure [27]. Furthermore, the synergistic effects of PTT and chemotherapy could be achieved by synthesizing drugloaded pDA nanoparticles: the acidic nature of the tumor environment triggers the release of anticancer drugs such as DOX and 7-ethyl-10-hydroxycamptothecin (SN38), and the simultaneous hypothermia caused by light-excited pDA could successfully suppress tumor growth in a tumor xenograft model [28].

\section{Drug delivery}

Currently, nanotechnology for drug delivery has received great attention because it allows for personalized healthcare by improving the targeting ability and drug loading efficiency to improve the therapeutic efficacy and reduce the side effects [69]. Therefore, a variety of nano-carriers with different sizes, structures and surface properties have been designed for smart drug delivery to date [69].
Among them, researchers have witnessed that melaninlike nanoparticles have many advantages in terms of their drug loading efficiency due to the strong $\pi-\pi$ interactions between the aromatic rings on drugs and melanin in addition to their biocompatibility and biodegradability [70]. Furthermore, a variety of molecules could be physically adsorbed or chemically immobilized on artificial melanin to achieve multifunctionality. Table 1 summarizes the use of melanin-like nanomaterials in smart drug delivery systems.

Melanin-like nanomaterials can act as a core template, a coating or an adhesive layer between the core and the outer materials. Ho and his colleague compared the drug-loading efficiency and release profile of a model anti-cancer drug, CPT, adsorbed on the surface of as-prepared pDA nanoparticles in a size ranging from $75 \mathrm{~nm}$ to $400 \mathrm{~nm}$, adjusted synthetically by $\mathrm{pH}$ [29]. As the size of the particles that were synthesized at a $\mathrm{pH}$ of approximately 7.5-8 increased, the drug loading efficiency also increased due to an advanced interior volume for the drugs of up to $11.81 \mu \mathrm{g}$ per $1 \mathrm{mg}$ of particles. In contrast to the loading efficiency, however, the drug release was much faster from the smaller particles, which were synthesized at $\mathrm{pH} 9$ [29]. Cui and his colleagues developed a $\mathrm{pH}$-dependent drug releasing platform by the synthesis of pDA hollow capsules decorated with DOX via a $\mathrm{pH}$-cleavable linker [32]. Over $85 \%$ of the DOX showed a sustained released up to $12 \mathrm{~h}$ at $\mathrm{pH} 5$, mimicking the tumor environment, whereas only $20 \%$ of DOX were released at neutral $\mathrm{pH}$ [32]. Targeting moieties could also be incorporated on the surface of artificial melanin templates with therapeutics for targeted drug delivery. W.-Q. Li et al. co-immobilized TPP with DOX on the surface of nanoparticles as the targeting moiety for mitochondria [30]. The developed nanoparticles can overcome drug resistance in long-term chemotherapy [30]. In addition, a gene delivery system was established by immobilization of amine-rich polymers such as polyethylenimine (PEI) on the surface of templated pDA NPs followed by complexation with plasmid DNA [31]. In this system, the toxic nature of PEI is suppressed by the conversion of the primary amine groups to secondary and tertiary amines during covalent conjugation with pDA [31].

Artificial melanin can also be an outer coating of nanomaterials to control drug release profiles and enhance biocompatibility and stability. A nano-thin coating layer of pDA on as-prepared insulin particles enabled $\mathrm{pH}$-responsive insulin release [33]. Sustained release of insulin was obtained up to $40 \mathrm{~h}$ at $\mathrm{pH} 7.4$, while only $29 \%$ was released at pH 5.4 [33]. W. Cheng et al. also employed pDA coating on DOX-loaded mesoporous silica nanoparticles as a $\mathrm{pH}$-sensitive gatekeeper [34]. They further immobilized folic acid on the surface- 
coated pDA for targeted cancer therapy [34]. S. Li et al. reported that pDA coating with casein could stabilize the zein-resveratrol nanocomplex against environmental stresses, including $\mathrm{pH}$, salinity, storage, redispersion, and UV irradiation, and enhance the antioxidant activity [71]. Finally, core-shell nanostructures have been synthesized by using artificial melanin-like pDA as an adhesive layer. M. Oroujeni et al. adopted pDA to immobilize the drug carrier 6-thio- $\beta$-cyclodextrin on the surface of magnetic nanoparticles, and hydrophobic diclofenac (DCF) was subsequently inserted into the surface-immobilized 6-thio- $\beta$-cyclodextrin [64]. Oligonucleotide-based aptamers and molecular probes have also been covalently immobilized on pDA-coated multifunctional nanoparticles via Michael addition reactions for potential applications in biosensors and nanomedicine [62].

\section{Bioimaging and theranostics}

Photoacoustic (PA) imaging is a noninvasive, nonionizing, and low-cost imaging method based on converting irradiated light energy absorbed by target molecules into thermal energy [28, 36]. Melanin and its derivatives, including pDA, have been suggested as endogenous chromophores that can produce PA signals in vivo [35]. Zhang et al. synthesized a melanin-loaded nano-liposome for PA imagingand MR imaging-guided photothermal cancer therapy, and the longitudinal relaxivity, $r_{1}$, of the synthesized nanoliposomes was $0.25 \mathrm{mM}^{-1} \mathrm{~s}^{-1}$ at $7 \mathrm{~T}$ [72]. Ju et al. reported that $\mathrm{pH}$-induced aggregation of melanin nanoparticles resulted in increased PA signal strength by generating overlapping thermal fields between aggregated nanoparticles [35]. In addition, various metal ions and reduced metals, targeting moieties, and therapeutic agents are allowed to be integrated with melanin-like nanoparticles for multimodal imaging and theranostics. Cationic poly-L-lysine-coated melanin nanoparticles could be used for anionic glycosaminoglycans (GAGs)-targeted PA imaging for the early diagnosis of articular cartilage degeneration in osteoarthritis [38]. Repenko et al. reported that melanin-coated gold nanomaterials with various shapes could be used as gastrointestinal imaging probes for PA imaging with excellent dispersibility, minimal cytotoxicity, and augmented PA responses [36].

In addition to PA imaging, organic/inorganic hybrid nanomaterials composed of melanin-like nanomaterials and inorganic metal ions have emerged for MR imaging-based theranostics. High loading efficiency of various metal ions on melanin-like nanomaterials can be achieved for improved contrast, and further incorporation of poly (ethylene glycol) (PEG) increases the circulation time in vivo. Ju and his colleagues reported that the longitudinal relaxivity, $r_{1}$, of $\mathrm{Fe}^{3+}$ loaded pDA nanoparticles was $17 \mathrm{mM}^{-1} \mathrm{~s}^{-1}$ at $3 \mathrm{~T}$, whereas that of the commercially available Gd-DOTA under the same conditions was $7.1 \mathrm{mM}^{-1} \mathrm{~s}^{-1}$ [73]. Similarly, $\mathrm{Gd}^{3+}$-loaded $13 \mathrm{~nm}$ sized natural melanin was synthesized for MRI with high stability in vivo and a high $r_{1}$ value [42]. Various metal ions, such as $\mathrm{Fe}^{3+}, \mathrm{Cu}^{2+}$ and $\mathrm{Mn}^{2+}$, could be loaded onto pDA nanoparticles for not only bioimaging but also simultaneous photothermal cancer therapy [39-41]; further co-immobilization of photosensitizers and therapeutic agents allows for multimodal theranostic applications. Wang et al. synthesized PEGylated-polydopamine (PDA) nanoparticles with the photosensitizer IR820 in addition to $\mathrm{Fe}^{3+}$ for MR imaging-guided combined photothermal and PDT [74]. pDA nanoparticles loaded with ICG, DOX, and $\mathrm{Mn}^{2+}$ could be used for MR imaging-guided combined chemotherapy and PTT in vivo [55]. Lin and his colleagues designed novel melanin-coated $\mathrm{Fe}_{3} \mathrm{O}_{4}$ nanoparticles with surface-adsorbed ${ }^{64} \mathrm{Cu}$ isotopes for multimodal imaging-guided cancer phototherapy [37]. The high brightness observed on T1-weighted images, the high affinity for metal ions, strong absorbance in the NIR region, and biocompatibility of natural melanin contributed to the MR imaging, PA imaging and photothermal imaging/therapy, and ${ }^{64} \mathrm{Cu}$ was employed for additional PET imaging [37].

\section{Fluorescence-based biosensing}

UV irradiation induces the autofluorescence of both natural melanin and synthetic melanin-like nanomaterials [75]. Therefore, the in-situ synthesis of these fluorescent nanomaterials could be an indicator in biosensing applications. Kong et al. developed a GSH sensing platform based on the $\mathrm{MnO}_{2}$-induced synthesis of fluorescent polydopamine nanoparticles (FPNPs) as an indicator in the absence of GSH, because GSH inhibits FPNP synthesis by reducing $\mathrm{MnO}_{2}$ to $\mathrm{Mn}^{2+}$ [76]. Similarly, they also detected ascorbic acid by using $\mathrm{CoOOH}$ as an oxidant to synthesize FPNPs instead of $\mathrm{MnO}_{2}$ because $\mathrm{CoOOH}$ is reduced to $\mathrm{CO}^{2+}$ in the presence of ascorbic acid and loses its activity to synthesize FPNPs [77].

Furthermore, chemical modification and/or partial degradation could enhance the intrinsic fluorescence intensity of as-prepared melanin-like nanoparticles. Gu and his colleagues synthesized tens of nanometer-sized FPNPs via ethylenediamine-induced partial degradation of as-prepared pDA nanoparticles [43]. The synthesized FPNPs were photostable and biocompatible, so they could be applied to the bioimaging of neuromast hair cells in the lateral line of zebrafish [43]. Similarly, Lin's group obtained FPNPs by using hydroxyl radicals instead of ethylenediamine because hydroxyl radicals can induce the partial degradation of as-prepared pDA nanoparticles by introducing hydroxyl groups onto the particles while reducing the $\pi-\pi$ interactions [78]. FPNPs were used for the detection of $\mathrm{Fe}^{3+}$ via electron-transfer- 
induced fluorescence quenching after the chelation of $\mathrm{Fe}^{3+}$ to the catechol groups of the FPNPs [78]. Interestingly, Yin et al. reported that further chemical reduction could enhance the fluorescence intensity of the asprepared FPNPs; they first synthesized FPNPs by sodium periodate-induced oxidation and then treated the FPNPs with sodium borohydride for the chemical reduction that tunes the surface of the as-prepared FPNPs to become full of hydroxyl groups [44]. The quantum yield of these FPNPs was $5.1 \%$, which is the highest rank among the reported FPNPs so far [44].

In addition to the intrinsic fluorescent properties of melanin-like nanomaterials, these nanomaterials can be applied in biosensing applications as fluorescence quenchers. Wang et al. synthesized mesoporous pDA nanoparticles and applied them as quenchers for fluorophore-conjugated single-strand DNA (ssDNA) probes to detect the downregulated let-7a and upregulated miRNA-21 in different types of cancer cells [45]. ssDNA probes were adsorbed on the surface of pDA nanoparticles through noncovalent bonds, such as $\pi-\pi$ stacking and electrostatic attraction, and then released from the surface by complexation with their complementary miRNAs, resulting in the recovery of their fluorescence [45]. Interestingly, mesoporous pDA nanoparticles successfully protected surface-bound ssDNA probes from cleavage by DNase I, which was used to target miRNA recycling for signal amplification [45]. Ma's group synthesized a novel upconversion@pDA core@shell nanoparticle with surface-complexed Cy3labeled aptamer probes for the detection of cytochrome $\mathrm{C}$ (Cyt C) in living cells. The fluorescence of the probes was quenched at the surface of the nanoparticles and then recovered by simultaneous dissociation and binding to Cyt $\mathrm{C}$ in the cytosol immediately after cellular uptake [45]. In addition, the steady upconversion luminescent signals from the core of the synthesized nanoparticles served for not only intracellular imaging but also as an internal standard for the quantitative measurement of Cyt C from Cy3-labeled aptamers [46].

\section{UV shielding in dermatology}

Melanin protects nearby cells in skin against sunlight by absorbing harmful ultraviolet (UV) rays and scavenging free radicals that cause DNA damage. Thus, synthetic melanin-like nanomaterials can be emerging as biocompatible sunscreens and next-generation therapeutics for people with melanin deficiency, such as albinism and vitiligo, which increases the risk of skin cancer [7]. Huang et al. demonstrated that melanin-like nanoparticles with a diameter of about $200 \mathrm{~nm}$ can be endocytosed, undergo perinuclear aggregation, and form a supranuclear cap in human epidermal keratinocytes, similar to natural melanosomes occurring in human skin
[7]. Accumulated melanin-like nanoparticles reduced reactive oxygen species (ROS) and prevented DNA damage in cells under UV irradiation [7]. C. Wang et al. suggested polydopamine-encapsulated polymeric gels as bioinspired sunscreens with superior UV shielding properties, nonphototoxicity, and nonirritating nature [79]. Similarly, Y. Wang et al. compared the UV shielding properties of polydopamine nanoparticles with natural melanin granules encapsulated in polymer matrix, indicating that decrease in the size of nanoparticles lead to increased UV-shielding and visible light transparency properties with reduced light scattering [80]. However, most of synthetic melanin-like nanomaterials up to date are brown-to-black due to the broad range absorbance from UV to near-IR range, which is a major obstacle in cosmetic applications. In addition to UV, absorbance manipulation in visible range of those materials can be the next-step to be achieved.

\section{Conclusion}

Melanin is a biopigments group in nature that possesses multifunctionality including ultraviolet protection, radical scavenging, and photothermal conversion. Its multifunctionality as well as intrinsic biocompatibility have inspired researchers to design novel melanin-like nanomaterials. Various enzymatic and chemical oxidation processes from natural precursors and their synthetic analogues have been reported, and additives can be easily incorporated in situ or form core-shell structures that expand the achievable scope of multifunctionality. Synthesized melanin-like nanomaterials have been emerging in various biomedical applications including therapy, bioimaging, and biosensing. In addition, the electrical conductivity and ion transport of melanin in nature have opened the very recent applications in soft bioelectronics. However, their bottom-up design/fabrication and tuning material properties at the molecular level are still limited because the chemical structure of these materials has not been fully elucidated thus far. Understanding the heterogeneity of the chemical structure and molecular weight distribution of oligomeric/polymeric building blocks and the driving force for nanoscale assembly via next-generation chemical and molecular-level analytical tools will be the next step forward to advance melaninbased biomedical applications in the future.

\footnotetext{
Abbreviations

Ce6: Chlorin e6; CPT: Camptothecin; Cyt C: Cytochrome C; DA: Dopamine; DCF: Hydrophobic diclofenac; DHI: 5,6-dihydroxyindole; DHICA: 5,6-

dihydroxyindole-2-carboxylic acid; DOX: Doxorubicin; FBGCs: Foreign body giant cells; FPNP: Fluorescent polydopamine nanoparticle;

GAG: Glycosaminoglycans; GSH: Glutathione; LPS: Lipopolysaccharide; MOF: Metal-organic framework; MRI: Magnetic resonance imaging; NIR: Nearinfrared; PA: Photoacoustic; PAl: Photoacoustic imaging; PDA: PEGylatedpolydopamine; pDA: Polydopamine; PDT: Photodynamic therapy; PEG: Poly (ethylene glycol); PEl: Polyethylenimine; PTT: Photothermal therapy;
} 
ROS: Reactive oxygen species; SN38: 7-ethyl-10-hydroxycamptothecin; ssDNA: Single-strand DNA; TPP: Triphenylphosphonium; UV: Ultraviolet

\section{Acknowledgements}

Not applicable.

\section{Authors' contributions}

The J. P, H. M and S. H wrote and improved the final manuscript. All authors read and approved the final manuscript.

\section{Funding}

This work was supported by the Basic Science Research Program through the National Research Foundation of Korea (NRF) funded by the Ministry of Education (NRF-2018R1D1A1B07045249 to S.H.) and the Cooperative Research Program for Agriculture Science and Accounts of Chemical Research Technology Development (Project PJ01323201 to S.H.) from the Rural Development Administration of the Republic of Korea.

\section{Availability of data and materials}

Not applicable.

\section{Ethics approval and consent to participate}

Not applicable.

\section{Consent for publication}

Not applicable.

\section{Competing interests}

The authors declare that they have no competing interests.

Received: 3 October 2019 Accepted: 25 November 2019 Published online: 03 December 2019

\section{References}

1. Costin GE, Hearing VJ. Human skin pigmentation: melanocytes modulate skin color in response to stress. FASEB J. 2007;21(4):976-94.

2. Goodman G, Bercovich D. Melanin directly converts light for vertebrate metabolic use: heuristic thoughts on birds, Icarus and dark human skin. Med Hypotheses. 2008;71(2):190-202.

3. Haining RL, Achat-Mendes C. Neuromelanin, one of the most overlooked molecules in modern medicine, is not a spectator. Neural Regen Res. 2017; 12(3):372-5.

4. Fedorow $H$, Tribl F, Halliday $G$, Gerlach M, Riederer P, Double KL. Neuromelanin in human dopamine neurons: comparison with peripheral melanins and relevance to Parkinson's disease. Prog Neurobiol. 2005;75(2): 109-24.

5. Kan H, Kim CH, Kwon HM, Park JW, Roh KB, Lee H, et al. Molecular control of phenoloxidase-induced melanin synthesis in an insect. J Biol Chem. 2008; 283(37):25316-23.

6. Lee H, Dellatore SM, Miller WM, Messersmith PB. Mussel-inspired surface chemistry for multifunctional coatings. Science. 2007;318(5849):426-30

7. Huang YR, Li YW, Hu ZY, Yue XJ, Proetto MT, Jones Y, et al. Mimicking melanosomes: polydopamine nanoparticles as artificial microparasols. ACS Cent Sci. 2017;3(6):564-9

8. Pillaiyar T, Namasivayam V, Manickam M, Jung SH. Inhibitors of Melanogenesis: an updated review. J Med Chem. 2018;61(17):7395-418.

9. Li F, Yu YY, Wang Q, Yuan JG, Wang P, Fan XR. Polymerization of dopamine catalyzed by laccase: comparison of enzymatic and conventional methods. Enzyme Microb Tech. 2018:119:58-64.

10. Lampel A, McPhee SA, Park HA, Scott GG, Humagain S, Hekstra DR, et al. Polymeric peptide pigments with sequence-encoded properties. Science. 2017;356(6342):1064-8.

11. Strube OI, Bungeler A, Bremser W. Enzyme-mediated in situ synthesis and deposition of nonaggregated melanin protoparticles. Macromol Mater Eng. 2016;301(7):801-4

12. Wang $X H$, Chen Z, Yang P, Hu JF, Wang Z, Li YW. Size control synthesis of melanin-like polydopamine nanoparticles by tuning radicals. Polym Chem. 2019:10(30):4194-200.

13. Della Vecchia NF, Luchini A, Napolitano A, D'Errico G, Vitiello G, Szekely N, et al. Tris buffer modulates polydopamine growth, aggregation, and paramagnetic properties. Langmuir. 2014;30(32):9811-8.
14. Jiang XL, Wang YL, Li MG. Selecting water-alcohol mixed solvent for synthesis of polydopamine nano-spheres using solubility parameter. Sci Rep. 2014;4:6070

15. Kim DJ, Ju KY, Lee JK. The synthetic melanin nanoparticles having an excellent binding capacity of heavy metal ions. B Korean Chem Soc. 2012; 33(11):3788-92.

16. Ponzio F, Barthes J, Bour J, Michel M, Bertani P, Hemmerle J, et al. Oxidant control of polydopamine surface chemistry in acids: a mechanism-based entry to superhydrophilic-superoleophobic coatings. Chem Mater. 2016; 28(13):4697-705

17. You I, Jeon H, Lee K, Do M, Seo YC, Lee HA, et al. Polydopamine coating in organic solvent for material-independent immobilization of water-insoluble molecules and avoidance of substrate hydrolysis. J Ind Eng Chem. 2017:46: 379-85

18. Ju KY, Lee $Y$, Lee S, Park SB, Lee JK. Bioinspired polymerization of dopamine to generate melanin-like nanoparticles having an excellent free-radicalscavenging property. Biomacromolecules. 2011:12(3):625-32.

19. d'Ischia M, Napolitano A, Pezzella A, Meredith P, Sarna T. Chemical and structural diversity in eumelanins: unexplored bio-optoelectronic materials. Angew Chem Int Ed. 2009;48(22):3914-21.

20. Kaxiras E, Tsolakidis A, Zonios G, Meng S. Structural model of eumelanin. Phys Rev Lett. 2006;97(21):218102.

21. Hong S, Na YS, Choi S, Song IT, Kim WY, Lee H. Non-covalent self-assembly and covalent polymerization co-contribute to polydopamine formation. Adv Funct Mater. 2012;22(22):4711-7.

22. Hong S, Wang Y, Park SY, Lee H. Progressive fuzzy cation- $\pi$ assembly of biological catecholamines. Sci Adv. 2018;4(9):eaat7457.

23. Chen CT, Martin-Martinez FJ, Jung GS, Buehler MJ. Polydopamine and eumelanin molecular structures investigated with ab initio calculations. Chem Sci. 2017:8(2):1631-41.

24. Zhao H, Zeng ZD, Liu L, Chen JW, Zhou HT, Huang LL, et al. Polydopamine nanoparticles for the treatment of acute inflammation-induced injury. Nanoscale. 2018;10(15):6981-91.

25. Bao XF, Zhao JH, Sun J, Hu M, Yang XR. Polydopamine nanoparticles as efficient scavengers for reactive oxygen species in periodontal disease. ACS Nano. 2018:12(9):8882-92.

26. Dong ZL, Feng LZ, Hao Y, Chen MC, Gao M, Chao Y, et al. Synthesis of hollow biomineralized $\mathrm{CaCO}_{3}$-polydopamine nanoparticles for multimodal imaging-guided cancer photodynamic therapy with reduced skin photosensitivity. J Am Chem Soc. 2018;140(6):2165-78.

27. Liu YL, Ai KL, Liu JH, Deng M, He YY, Lu LH. Dopamine-melanin colloidal nanospheres: an efficient near-infrared photothermal therapeutic agent for in vivo cancer therapy. Adv Mater. 2013;25(9):1353-9.

28. Wang XY, Zhang JS, Wang YT, Wang CP, Xiao JR, Zhang Q, et al. Multiresponsive photothermal-chemotherapy with drug-loaded melanin-like nanoparticles for synergetic tumor ablation. Biomaterials. 2016:81:114-24

29. Ho CC, Ding SJ. The pH-controlled nanoparticles size of polydopamine for anti-cancer drug delivery. J Mater Sci Mater Med. 2013;24(10):2381-90.

30. Li WQ, Wang ZG, Hao SJ, He HZ, Wan Y, Zhu CD, et al. Mitochondriatargeting polydopamine nanoparticles to deliver doxorubicin for overcoming drug resistance. ACS Appl Mater Interfaces. 2017;9(20):16794803.

31. Priyam A, Nagar P, Sharma AK, Kumar P. Mussel-inspired polydopaminepolyethylenimine conjugated nanoparticles as efficient gene delivery vectors for mammalian cells. Colloid Surface B. 2018;161:403-12.

32. Cui JW, Yan Y, Such GK, Liang K, Ochs CJ, Postma A, et al. Immobilization and intracellular delivery of an anticancer drug using mussel-inspired polydopamine capsules. Biomacromolecules. 2012;13(8):2225-8.

33. Li H, Jia $Y$, Feng $X Y$, Li JB. Facile fabrication of robust polydopamine microcapsules for insulin delivery. J Colloid Interface Sci. 2017:487:12-9.

34. Cheng $W$, Nie JP, Xu L, Liang CY, Peng Y, Liu G, et al. pH-sensitive delivery vehicle based on folic acid-conjugated polydopamine-modified mesoporous silica nanoparticles for targeted cancer therapy. ACS App Mater Interfaces. 2017;9(22):18462-73

35. Ju KY, Kang J, Pyo J, Lim J, Chang JH, Lee JK. pH-induced aggregated melanin nanoparticles for photoacoustic signal amplification. Nanoscale. 2016:8(30):14448-56.

36. Repenko T, Rix A, Nedilko A, Rose J, Hermann A, Vinokur R, et al. Strong photoacoustic signal enhancement by coating gold nanoparticles with melanin for biomedical imaging. Adv Funct Mater. 2018;28(7):1705607. 
37. Lin J, Wang $M, H u H$, Yang XY, Wen B, Wang ZT, et al. Multimodal-imagingguided cancer phototherapy by versatile biomimetic theranostics with UV and gamma-irradiation protection. Adv Mater. 2016;28(17):3273-9.

38. Chen L, Ji Y, Hu XM, Cui C, Liu H, Tang YF, et al. Cationic poly-L-lysineencapsulated melanin nanoparticles as efficient photoacoustic agents targeting to glycosaminoglycans for the early diagnosis of articular cartilage degeneration in osteoarthritis. Nanoscale. 2018;10(28):13471-84.

39. Ge R, Lin M, Li X, Liu SW, Wang WJ, Li SY, et al. Cu+-loaded polydopamine nanoparticles for magnetic resonance imaging-guided $\mathrm{pH}$ - and nearinfrared-light-stimulated thermochemotherapy. ACS Appl Mater Interfaces. 2017;9(23):19706-16.

40. Miao ZH, Wang H, Yang H, Li ZL, Zhen L, Xu CY. Intrinsically $\mathrm{Mn}^{2+}$-chelated polydopamine nanoparticles for simultaneous magnetic resonance imaging and photothermal ablation of cancer cells. ACS Appl Mater Interfaces. 2015; 7(31):16946-52.

41. Liu FY, He XX, Zhang JP, Chen HD, Zhang HM, Wang ZX. Controllable synthesis of polydopamine nanoparticles in microemulsions with $\mathrm{pH}$ activatable properties for cancer detection and treatment. J Mater Chem B. 2015;3(33):6731-9.

42. Hong SH, Sun Y, Tang C, Cheng K, Zhang RP, Fan QL, et al. Chelator-free and biocompatible melanin nanoplatform with facile loading gadolinium and copper-64 for bioimaging. Bioconjug Chem. 2017;28(7):1925-30.

43. Gu GE, Park CS, Cho HJ, Ha TH, Bae J, Kwon OS, et al. Fluorescent polydopamine nanoparticles as a probe for zebrafish sensory hair cells targeted in vivo imaging. Sci Rep. 2018;8:4393.

44. Yin HG, Zhang KL, Wang LJ, Zhou K, Zeng J, Gao D, et al. Redox modulation of polydopamine surface chemistry: a facile strategy to enhance the intrinsic fluorescence of polydopamine nanoparticles for sensitive and selective detection of Fe ${ }^{3+}$. Nanoscale. 2018;10(37):18064-73.

45. Wang ZQ, Zhang JX, Chen F, Cai KY. Fluorescent miRNA analysis enhanced by mesopore effects of polydopamine nanoquenchers. Analyst. 2017; 142(15):2796-804.

46. Ma L, Liu FY, Lei Z, Wang ZX. A novel upconversion@polydopamine core@shell nanoparticle based aptameric biosensor for biosensing and imaging of cytochrome c inside living cells. Biosens Bioelectron. 2017;87: $638-45$.

47. Chen MH, Wen Q, Gu FL, Gao JW, Zhang CC, Wang QM. Mussel chemistry assembly of a novel biosensing nanoplatform based on polydopamine fluorescent dot and its photophysical features. Chem Eng J. 2018;342:331-8.

48. Ang JM, Du YH, Tay BY, Zhao CY, Kong JH, Stubbs LP, et al. One-pot synthesis of Fe (III)-Polydopamine complex nanospheres: morphological evolution, mechanism, and application of the carbonized hybrid nanospheres in catalysis and Zn-air battery. Langmuir. 2016;32(36):9265-75.

49. Wang DD, Wu HH, Zhou JJ, Xu PP, Wang CL, Shi RH, et al. In situ one-pot synthesis of MOF-polydopamine hybrid nanogels with enhanced photothermal effect for targeted cancer therapy. Adv Sci. 2018;5(6):1800287.

50. Gao Y, Wu X, Zhou L, Su Y, Dong CM. A sweet polydopamine nanoplatform for synergistic combination of targeted chemo-photothermal therapy. Macromol Rapid Commun. 2015:36(10):916-22.

51. Luo HY, Gu CW, Zheng WH, Dai F, Wang XL, Zheng Z. Facile synthesis of novel size-controlled antibacterial hybrid spheres using silver nanoparticles loaded with poly-dopamine spheres. RSC Adv. 2015;5(18):13470-7.

52. Fan QL, Cheng K, Hu X, Ma XW, Zhang RP, Yang M, et al. Transferring biomarker into molecular probe: melanin nanoparticle as a naturally active platform for multimodality imaging. J Am Chem Soc

2014;136(43):15185-94.

53. Li YY, Jiang $C H$, Zhang DW, Wang $Y$, Ren $X Y$, Ai KL, et al. Targeted polydopamine nanoparticles enable photoacoustic imaging guided chemophotothermal synergistic therapy of tumor. Acta Biomater. 2017:47:124-34.

54. Jiao L, Xu Z, Du W, Li H, Yin M. Fast preparation of polydopamine nanoparticles catalyzed by $\mathrm{Fe}^{2+} / \mathrm{H}_{2} \mathrm{O}_{2}$ for visible sensitive smartphoneenabled cytosensing. ACS Appl Mater Interfaces. 2017;9(34):28339-45.

55. Dong ZL, Gong H, Gao M, Zhu WW, Sun XQ, Feng LZ, et al. Polydopamine nanoparticles as a versatile molecular loading platform to enable imagingguided cancer combination therapy. Theranostics. 2016;6(7):1031-42.

56. Wang $X L$, Wang $N$, Yang $Y$, Wang $X X$, Liang JY, Tian XX, et al. Polydopamine nanoparticles carrying tumor cell lysate as a potential vaccine for colorectal cancer immunotherapy. Biomater Sci Uk. 2019;7(7):3062-75.

57. Yavuz E, Tokalioglu S, Patat S. Core-shell $\mathrm{Fe}_{3} \mathrm{O}_{4}$ polydopamine nanoparticles as sorbent for magnetic dispersive solid-phase extraction of copper from food samples. Food Chem. 2018;263:232-9.
58. Cheng YX, Zhang SP, Kang N, Huang JP, LV XL, Wen K, et al. Polydopaminecoated manganese carbonate nanoparticles for amplified magnetic resonance imaging-guided photothermal therapy. ACS Appl Mater Interfaces. 2017:9(22):19296-306.

59. Liu XS, Cao JM, Li H, Li JY, Jin Q, Ren KF, et al. Mussel-inspired polydopamine: a biocompatible and ultrastable coating for nanoparticles in vivo. ACS Nano. 2013;7(10):9384-95.

60. Hong S, Kim KY, Wook HJ, Park SY, Lee KD, Lee DY, et al. Attenuation of the in vivo toxicity of biomaterials by polydopamine surface modification. Nanomedicine Uk. 2011;6(5):793-801.

61. Tao W, Zeng XW, Wu J, Zhu X, Yu XH, Zhang XD, et al. Polydopamine-based surface modification of novel nanoparticle-aptamer bioconjugates for in vivo breast cancer targeting and enhanced therapeutic effects. Theranostics. 2016;6(4):470-84.

62. Wang CX, Zhou JJ, Wang P, He WS, Duan HW. Robust nanoparticle-DNA conjugates based on mussel-inspired polydopamine coating for cell imaging and tailored self-assembly. Bioconjug Chem. 2016;27(3):815-23.

63. Zhu DW, Tao W, Zhang HL, Liu G, Wang T, Zhang LH, et al. Docetaxel (DTX)loaded polydopamine-modified TPGS-PLA nanoparticles as a targeted drug delivery system fore the treatment of liver cancer. Acta Biomater. 2016;30: 144-54.

64. Oroujeni M, Kaboudin B, Xia W, Jonsson P, Ossipov DA. Conjugation of cyclodextrin to magnetic $\mathrm{Fe}_{3} \mathrm{O}_{4}$ nanoparticles via polydopamine coating for drug delivery. Prog Org Coat. 2018;114:154-61.

65. Nieto C, Vega MA, Marcelo G, Valle EM. Polydopamine nanoparticles kill cancer cells. RSC Adv. 2018;8(63):36201.

66. Han J, Park W, Park SJ, Na K. Photosensitizer-conjugated hyaluronic acidshielded polydopamine nanoparticles for targeted photomediated tumor therapy. ACS Appl Mater Interfaces. 2016;8(12):7739-47.

67. Tran ML, Powell BJ, Meredith P. Chemical and structural disorder in eumelanins: a possible explanation for broadband absorbance. Biophys J. 2006:90(3):743-52

68. Zou LL, Wang H, He B, Zeng LJ, Tan T, Cao HQ, et al. Current approaches of photothermal therapy in treating cancer metastasis with nanotherapeutics. Theranostics. 2016;6(6):762-72.

69. Din FU, Aman W, Ullah I, Qureshi OS, Mustapha O, Shafique S, et al. Effective use of nanocarriers as drug delivery systems for the treatment of selected tumors. Int J Nanomedicine. 2017:12:7291-309.

70. Liu FY, He XX, Lei Z, Liu L, Zhang JP, You HP, et al. Facile preparation of doxorubicin-loaded upconversion@polydopamine nanoplatforms for simultaneous in vivo multimodality imaging and chemophotothermal synergistic therapy. Adv Healthc Mater. 2015;4(4):559-68.

71. Li SM, Li ZX, Pang JF, Chen J, Wang HD, Xie QL, et al. Polydopaminemediated carrier with stabilizing and self-antioxidative properties for polyphenol delivery systems. Ind Eng Chem Res. 2018;57(2):590-9.

72. Zhang L, Sheng DL, Wang D, Yao YZ, Yang K, Wang ZG, et al. Bioinspired multifunctional melanin-based nanoliposome for photoacoustic/magnetic resonance imaging-guided efficient photothermal ablation of cancer. Theranostics. 2018;8(6):1591-606.

73. Ju KY, Lee JW, Im GH, Lee S, Pyo J, Park SB, et al. Bio-inspired, melanin-like nanoparticles as a highly efficient contrast agent for $\mathrm{T}_{1}$-weighted magnetic resonance imaging. Biomacromolecules. 2013;14(10):3491-7.

74. Wang JJ, Guo Y, Hu J, Li WC, Kang YJ, Cao Y, et al. Development of multifunctional polydopamine nanoparticles as a theranostic nanoplatform against cancer cells. Langmuir. 2018;34(32):9516-24.

75. Elleder M, Borovansky J. Autofluorescence of melanins induced by ultraviolet radiation and near ultraviolet light. A histochemical and biochemical study. Histochem J. 2001;33(5):273-81.

76. Kong XJ, Wu S, Chen TT, Yu RQ, Chu X. MnO -induced synthesis of fluorescent polydopamine nanoparticles for reduced glutathione sensing in human whole blood. Nanoscale. 2016:8(34):15604-10.

77. Zhao YY, Li L, Yu RQ, Chen TT, Chu X. CoOOH-induced synthesis of fluorescent polydopamine nanoparticles for the detection of ascorbic acid. Anal Methods. 2017;9(37):5518-24.

78. Lin JH, Yu CJ, Yang YC, Tseng WL. Formation of fluorescent polydopamine dots from hydroxyl radical-induced degradation of polydopamine nanoparticles. Phys Chem Chem Phys. 2015;17(23):15124-30.

79. Wang CP, Wang D, Dai TJ, Xu P, Wu P, Zou Y, et al. Skin pigmentationinspired polydopamine sunscreens. Adv Funct Mater. 2018;28(33): 1802127. 
80. Wang Y, Wang XF, Li T, Ma PM, Zhang SW, Du ML, et al. Effects of melanin on optical behavior of polymer: from natural pigment to materials applications. ACS Appl Mater Interfaces. 2018;10(15):13100-6.

\section{Publisher's Note}

Springer Nature remains neutral with regard to jurisdictional claims in published maps and institutional affiliations.

Ready to submit your research? Choose BMC and benefit from:

- fast, convenient online submission

- thorough peer review by experienced researchers in your field

- rapid publication on acceptance

- support for research data, including large and complex data types

- gold Open Access which fosters wider collaboration and increased citations

- maximum visibility for your research: over $100 \mathrm{M}$ website views per year

At BMC, research is always in progress. 